\title{
Outcomes of Patients Hospitalized With Community-Acquired, Health Care-Associated, and Hospital-Acquired Pneumonia
}

Mario Venditti, MD; Marco Falcone, MD; Salvatore Corrao, MD; Giuseppe Licata, MD; Pietro Serra, MD; and the Study Group of the Italian Society of Internal Medicine*

Background: Traditionally, pneumonia has been classified as either community- or hospital-acquired. Although only limited data are available, health care-associated pneumonia has been recently proposed as a new category of respiratory infection. "Health careassociated pneumonia" refers to pneumonia in patients who have recently been hospitalized, had hemodialysis, or received intravenous chemotherapy or reside in a nursing home or long-term care facility.

Objective: To ascertain the epidemiology and outcome of community-acquired, health care-associated, and hospital-acquired pneumonia in adults hospitalized in internal medicine wards.

Design: Multicenter, prospective observational study.

Setting: 55 hospitals in Italy comprising 1941 beds.

Patients: 362 patients hospitalized with pneumonia during two 1-week surveillance periods.

Measurements: Cases of radiologically and clinically assessed pneumonia were classified as community-acquired, health careassociated, or hospital-acquired and rates were compared.

Results: Of the 362 patients, $61.6 \%$ had community-acquired pneumonia, $24.9 \%$ had health care-associated pneumonia, and $13.5 \%$ had hospital-acquired pneumonia. Patients with health careassociated pneumonia had higher mean Sequential Organ Failure Assessment scores than did those with community-acquired pneumonia (3.0 vs. 2.0 ), were more frequently malnourished $(11.1 \%$ vs. $4.5 \%$, and had more frequent bilateral (34.4\% vs. $19.7 \%$ ) and multilobar (27.8\% vs. $21.5 \%$ ) involvement on a chest radiograph. Patients with health care-associated pneumonia also had higher fatality rates $(17.8 \%[\mathrm{Cl}, 10.6 \%$ to $24.9 \%]$ vs. $6.7 \%[\mathrm{Cl}, 2.9 \%$ to $10.5 \%]$ ) and longer mean hospital stay (18.7 days [Cl, 15.9 to 21.5 days] vs. 14.7 days [Cl, 13.4 to 15.9 days]). Logistic regression analysis revealed that depression of consciousness (odds ratio [OR], $3.2[\mathrm{Cl}, 1.06$ to 9.8]), leukopenia (OR, $6.2[\mathrm{Cl}, 1.01$ to 37.6]), and receipt of empirical antibiotic therapy not recommended by international guidelines $(\mathrm{OR}, 6.4[\mathrm{Cl}, 2.3$ to 17.6]) were independently associated with increased intrahospital mortality.

Limitations: The number of patients with health care-associated pneumonia was relatively small. Microbiological investigations were not always homogeneous. The study included only patients with pneumonia that required hospitalization; results may not apply to patients treated as outpatients.

Conclusion: Health care-associated pneumonia should be considered a distinct subset of pneumonia associated with more severe disease, longer hospital stay, and higher mortality rates. Physicians should differentiate between patients with health care-associated pneumonia and those with community-acquired pneumonia and provide more appropriate initial antibiotic therapy.

Funding: None.

Ann Intern Med. 2009;150:19-26.

www.annals.org

For author affiliations, see end of text.

* For a complete list of study group members, see the Appendix (available at www.annals.org).
P neumonia represents a spectrum of diseases that range from community-acquired to hospital-acquired and ventilator-associated pneumonia. Despite advances in diagnosis, antimicrobial therapy, and supportive care, pneumonia remains an important cause of morbidity and mortality, especially in patients who require hospitalization (1).

In recent years, changes in the health care system have shifted a considerable part of patient care from hospitals to the community. As a result, the traditional distinction between community- and hospital-acquired infections has become less clear. Infections occurring among outpatients in contact with the health care system have been termed "health care-associated infections" (2).

The 2005 American Thoracic Society and Infectious Diseases Society of America guidelines (3) propose a new category of pneumonia: health care-associated pneumonia. This category includes patients with pneumonia who have had recent contact with the health care system through nursing homes, hemodialysis clinics, or hospitalization (3, 4). However, limited data exist to validate this proposal. The largest studies supporting the proposal are 2 retrospec- tive analyses of patients with culture-positive pneumonia admitted to U.S. hospitals $(5,6)$ and a recent prospective single-center study from Barcelona, Spain (7). In the latter study, Carratalà and colleagues observed that a substantial number of patients admitted to the emergency department with pneumonia had health care-associated pneumonia (7). However, the results of these 3 studies differ in several respects, probably because of differences in study design, patient populations, and methodology.

See also:

Print

Editors' Notes. . . . . . . . . . . . . . . . . . . . . . 20

Summary for Patients. . . . . . . . . . . . . . . . I-36

Web-Only

Appendix

Appendix Tables

Conversion of graphics into slides 
ArTICLE $\mid$ Health Care-Associated Pneumonia as a Distinct Subset of Pneumonia

\section{Context}

Pneumonia in patients who were recently hospitalized, reside in a long-term health facility, or are receiving hemodialysis or intravenous chemotherapy is known as "health care-associated pneumonia." Health care-associated pneumonia seems to differ from pneumonia that occurs in patients without these characteristics.

\section{Contribution}

This study of 362 patients hospitalized with pneumonia showed that the $25 \%$ who had health care-associated pneumonia had more severe clinical courses than those with community-acquired pneumonia and a mortality rate close to that of patients with hospital-acquired pneumonia. Receipt of antibiotics not recommended by guidelines was associated with death from health care-associated pneumonia.

\section{Caution}

These findings may not apply to pneumonia that does not require hospitalization.

- The Editors

We sought to ascertain the epidemiology and outcome of community-acquired, health care-associated, and hospital-acquired pneumonia in patients hospitalized in Italian internal medicine wards and to better characterize these clinical entities in a large, national cohort.

\section{Methods}

\section{Setting and Study Period}

This cohort study, open to all members of the Italian Society of Internal Medicine, was performed prospectively in 59 divisions of internal medicine in 55 Italian hospitals (18 teaching and 37 community hospitals, comprising 1941 beds). All members of the Italian Society of Internal Medicine were informed about the study by e-mail or telephone (the protocol and design of the study were also available on the Society's Web site, www.simi.it, and in its official journal). Participation was free and spontaneous. As shown in Figure 1, participating centers were geographically distributed across most regions of Italy. Cases of pneumonia were assessed during 2 active 1-week surveillance periods (22 to 29 January 2007 and 25 June to 2 July 2007). The study included adults ( $>18$ years) already hospitalized with pneumonia and those who were newly admitted during the study periods (incident cases). An institutional review board of the Board of the Italian Society of Internal Medicine approved this study.

\section{Patients}

Our case definition was radiologic evidence of pneumonia and at least 2 of the following criteria: fever or hypothermia (temperature $>38^{\circ} \mathrm{C}$ or $<35^{\circ} \mathrm{C}$ ), dyspnea, cough and purulent sputum, pleuritic chest pain, or signs of consolidation on respiratory auscultation. Our definition of hospital-acquired pneumonia also included the absence of pulmonary infiltrates on a chest radiograph at the time of hospital admission.

We classified patients as having health care-associated pneumonia if they had attended a hospital or hemodialysis clinic or received intravenous chemotherapy in the past 30 days, had been admitted to an acute-care hospital for at least 2 days or had surgery in the past 180 days, or resided in a nursing home or long-term care facility. We classified patients as having hospital-acquired pneumonia if they received their diagnosis after being hospitalized for more than 72 hours or within 10 days of leaving the hospital. We considered patients who fulfilled the criteria for health care-associated pneumonia but developed pneumonia in the 10 days after discharge as having hospital-acquired pneumonia. We classified patients as having communityacquired pneumonia if they did not fit the criteria for either health care-associated or hospital-acquired pneumonia.

Exclusion criteria included acquisition of pneumonia in the intensive care unit or in another hospital and HIV infection.

\section{Measurements}

To stratify patients into risk classes, we used the prediction rule calculated according to the Pneumonia Severity Index and the CURB-65 (Confusion, Urea nitrogen, Respiratory rate, Blood pressure, and age 65 years or older) score $(8,9)$.

We recorded the following data: age; sex; intrinsic and extrinsic risk factors, such as underlying diseases, pharma-

\section{Figure 1. Geographic distribution of participating centers.}

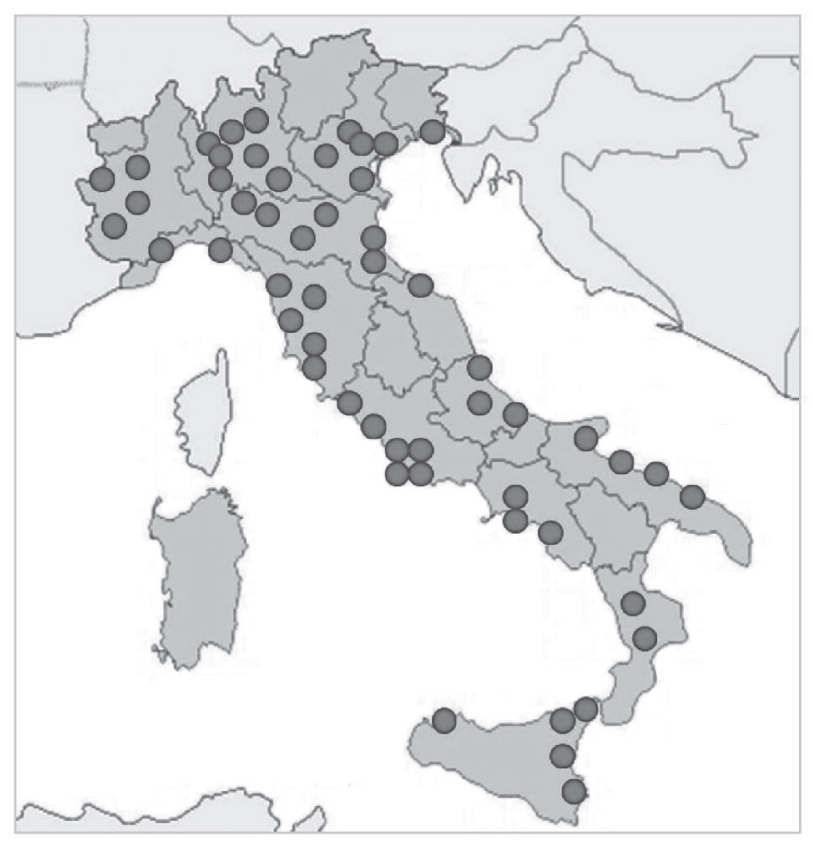


cologic immunosuppression (steroids and cytostatic drugs), leukopenia, previous antibiotic therapy, use of histamine-2 blockers or antacids, nebulization, previous invasive techniques (such as endotracheal intubation or tracheotomy), surgery, or malnutrition; clinical signs and symptoms, such as cough, dyspnea, chest pain, body temperature, respiratory rate, heart rate, arterial systolic and diastolic blood pressure, and depression of consciousness; results of laboratory studies, such as blood gas analysis, leukocyte count, platelet count, serum creatinine level, and total and fractionated bilirubin; chest radiography pattern, such as lateral or bilateral involvement, number of lobes affected, and presence of pleural effusion; results of microbiological studies, such as sputum culture, blood culture, urinary antigen detection, pleural fluid culture, and bronchoalveolar lavage culture; antibiotic therapy; and outcome. We also recorded in-hospital complications and deaths. At the clinical end points of hospital discharge or death, we retrieved data on in-hospital death, length of hospital stay, and adequacy of initial antimicrobial treatment.

We calculated the prevalence of pneumonia by dividing the number of patients hospitalized with pneumonia on the first day of the study by the total number of patients hospitalized on that day. We considered underlying diseases to be comorbid conditions. We defined malnutrition as a body mass index less than $20 \mathrm{~kg} / \mathrm{m}^{2}$ for men and less than $18.5 \mathrm{~kg} / \mathrm{m}^{2}$ for women (10). We defined depression of consciousness as any alteration in the level of alertness observed at the time of presentation of pneumonia or in the previous 72 hours. Severity of clinical conditions was assessed at the time of presentation by using the Sequential Organ Failure Assessment score. We considered extrinsic risk factors if they were present or occurred within 30 days before the diagnosis of pneumonia. We defined previous antibiotic therapy as receipt of any antibiotic for more than 48 hours during the previous 3 months (11).

\section{Antimicrobial Treatment Evaluation}

We defined empirical antibiotic therapy as antibiotics administered on the first day of therapy for pneumonia and considered an empirical antibiotic regimen as adherent to guidelines if it was concordant with the available American Thoracic Society and Infectious Diseases Society of America guidelines for community-acquired, hospital-acquired, or health care-associated pneumonia $(3,12)$. We analyzed the appropriateness of antibiotic therapy for all patients with an etiologic diagnosis according to susceptibility test criteria for lower respiratory tract pathogens. We rated an antimicrobial treatment as inadequate if 1 or more of the organisms present were known to have intrinsic resistance or were found to be resistant through susceptibility testing. For cases in which Pseudomonas aeruginosa was isolated, we considered treatment adequate only if the patient received combination treatment with at least 2 drugs to which the strain was fully susceptible (13).

\section{Statistical Analysis}

Data are presented as mean values $(95 \% \mathrm{CI})$ for quantitative variables and as relative frequencies $(95 \% \mathrm{CI})$ for categorical variables. We performed nonparametric or equivalent tests for group comparison of quantitative variables: the Mann-Whitney $U$ test for 2-group comparisons, 1 -way analysis of variance on the ranks of observations for 3 -group mean value comparisons, and post hoc Bonferroni correction for pairwise comparisons. We used the Pearson chi-square test and the generalized Fisher exact test for contingency table analysis. We investigated the association among pneumonia classes (as a dependent multinomial 3-level variable) and the other categorical variables by using multinomial logistic regression. After bivariate analyses, we selected independent variables at a $P$ value less than 0.100 . All were put into a model of multivariate multinomial logistic regression analysis. We used the community-acquired pneumonia category as a reference for comparisons. Finally, we performed logistic regression for binary outcomes to analyze the association between variables and mortality and used the Hosmer-Lemeshow methodology for variable selection (14). We then put all the selected variables into the multivariate model. We performed the Pearson goodness-of-fit test to assess the overall fit of the model. We computed odds ratios (ORs), derived from the covariates, and confidence intervals. We used a bootstrap procedure to resample observations (1000 replications per time) according to hospital ward clusters for both descriptive and inference analyses. A 2 -tailed $P$ value less than 0.050 was considered statistically significant. We used STATA/SE, version 9.2 for Windows (StataCorp, College Station, Texas), to analyze the data.

\section{Role of the Funding Source}

Our study received no external funding.

\section{RESULTS \\ Patient Characteristics}

Figure 2 shows the flow of study participants. The sample included 362 patients with pneumonia-271 hospitalized during the first week and 91 during the second week of study. The number of cases detected in each center ranged from 1 to 26 . The prevalence of pneumonia was $11.9 \%$ (CI, $11.2 \%$ to $12.6 \%$ ) during the winter period (22 to 29 January 2007 ) and $4.7 \%$ (CI, $4.2 \%$ to $5.2 \%$ ) during the summer period (25 June to 2 July 2007).

Most patients (60.2\%) were men, and the mean age was 75.5 years. The most frequently encountered comorbid conditions were chronic obstructive pulmonary disease $(36.2 \%)$, heart failure $(30.7 \%)$, dementia $(24 \%)$, diabetes mellitus (21\%), renal failure (20.7\%), and cancer (18.8\%). At the time of diagnosis of pneumonia, 176 (48.6\%) patients had at least 2 comorbid conditions and $80(22.1 \%)$ had more than 2 comorbid conditions. Overall, 223 (61.6\%) patients had community-acquired pneumonia, 90 (24.9\%) had health care-associated pneumonia, and 49

6 January 2009 Annals of Internal Medicine $\mid$ Volume 150 - Number $1 \mid \mathbf{2 1}$ 
Figure 2. Study flow diagram.

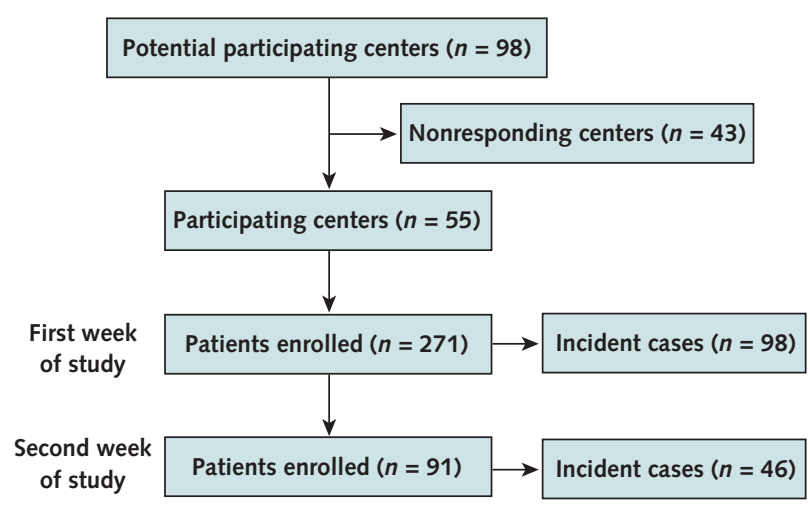

(13.5\%) had hospital-acquired pneumonia. First- and second-week patient populations did not significantly differ in terms of demographic characteristics, comorbid conditions, category of pneumonia, or outcome (data not shown).

Tables 1 and 2 compare the characteristics of patients with community-acquired pneumonia, health careassociated pneumonia, and hospital-acquired pneumonia. Table 3 lists the criteria for inclusion in the health careassociated pneumonia group. Appendix Tables 1, 2, and 3 (available at www.annals.org) show the analysis of incident cases only.

Compared with patients with community-acquired pneumonia, those with health care-associated pneumonia were more commonly in high-risk pneumonia severity index classes (Table 1), had a higher incidence of malnutrition, and had more frequent bilateral involvement during radiographic examination (Table 2 ). In addition, patients with health care-associated pneumonia received gastric acidreducing agents or aerosolized drugs more frequently and were febrile at hospital admission less frequently than patients with community-acquired pneumonia. However, the 2 groups did not significantly differ in terms of mean age, sex, or the presence of comorbid conditions. At the time of diagnosis, patients with community-acquired pneumonia had significantly lower mean Sequential Organ Failure Assessment scores (2.0 [CI, 1.7 to 2.3]) than did patients with health care-associated (3.0 [CI, 2.6 to 3.4]) or hospitalacquired pneumonia (2.8 [CI, 2.4 to 3.2]).

A significantly higher percentage of patients with hospitalacquired pneumonia had leukopenia, multilobar infiltrates, a tracheostomy procedure, or a history of recent endotracheal intubation than did those with community-acquired or health care-associated pneumonia (Table 2).

We analyzed categorical variables by using a multivariate model that included age, length of hospital stay, duration of antibiotic therapy, etiologic diagnosis, presence of at least 2 comorbid conditions, malnutrition, therapy with histamine- 2 blockers or antacids, aerosolized drug therapy, fever, cough, bilateral or multilobar involvement at chest radiograph, monotherapy, antibiotic therapy adherent to international guidelines, appropriateness of antibiotic therapy, and death. Multinomial logistic regression showed that histamine-2 blocker or antacid administration (relative risk ratio $[\mathrm{RRR}], 3.3$ [CI, 1.6 to 7.1$] ; P=0.002$ ), hospital stay longer than 20 days (RRR, 2.7 [CI, 1.2 to $6.2 ; P=$ 0.010 ), and receipt of an empirical antibiotic not recommended by international guidelines (RRR, 4.1 [CI, 1.6 to 10.4$] ; P=0.004)$ were independently associated with health care-associated pneumonia (compared with community-acquired pneumonia). Histamine-2 blocker or antacid administration (RRR, 8.9 [CI, 2.3 to 34.5]; $P=0.002)$ and hospital stay longer than 20 days (RRR, 5.3 [CI, 2.2 to 12.4$] ; P<0.001)$ were independently associated with hospital-acquired pneumonia (compared with community-acquired pneumonia).

\section{Antimicrobial Therapy and Outcomes}

Table 4 shows data on antimicrobial therapy and outcomes of the 3 pneumonia groups. Overall, 189 (52.2\%) patients received an initial empirical therapy according to international guidelines. Patients with health care-associated pneumonia were more frequently treated with an empirical therapy that was not consistent with available guidelines $(P<0.001)$.

The mean duration of antibiotic therapy and length of hospital stay were also significantly higher in patients with health care-associated pneumonia than in those with community-acquired pneumonia. The mortality rate associated with community-acquired pneumonia

\section{Table 1. Risk Stratification}

\begin{tabular}{|c|c|c|c|}
\hline Variable & $\begin{array}{l}\text { Community-Acquired } \\
\text { Pneumonia }(95 \% \mathrm{CI}), \% \\
(n=223)\end{array}$ & $\begin{array}{l}\text { Health Care-Associated } \\
\text { Pneumonia }(95 \% \mathrm{Cl}), \% \\
(n=90)\end{array}$ & $P$ Value \\
\hline Mean PSI score & $110.6(106.4-114.8)$ & $121.8(117.1-126.4)$ & 0.004 \\
\hline PSI high-risk class* & $76.2(70.6-81.8)$ & $91.1(85.5-96.7)$ & $<0.001$ \\
\hline CURB-65 high-risk scoret & $15.7(10-21.4)$ & $30.0(19.5-40.5)$ & 0.010 \\
\hline
\end{tabular}

CURB-65 = Confusion, Urea nitrogen, Respiratory rate, Blood pressure, age 65 years or older; PSI = Pneumonia Severity Index.

* Class IV or V (8).

+ Class III (9). 
Health Care-Associated Pneumonia as a Distinct Subset of Pneumonia $\mid$ ARTICLE

Table 2. Patient Characteristics

\begin{tabular}{|c|c|c|c|c|}
\hline Characteristic & $\begin{array}{l}\text { Community- } \\
\text { Acquired } \\
\text { Pneumonia } \\
(n=223)\end{array}$ & $\begin{array}{l}\text { Health Care- } \\
\text { Associated } \\
\text { Pneumonia } \\
(n=90)\end{array}$ & $\begin{array}{l}\text { Hospital- } \\
\text { Acquired } \\
\text { Pneumonia } \\
(n=49)\end{array}$ & $P$ Value \\
\hline Men, \% & 58.8 & 62.2 & 63.3 & 0.78 \\
\hline Mean age, $y$ & 73.9 & 77.4 & 79.9 & 0.060 \\
\hline Chronic obstructive pulmonary disease, $\%$ & 39.0 & 30.0 & 34.7 & 0.45 \\
\hline Heart failure, \% & 27.8 & 37.7 & 30.6 & 0.25 \\
\hline Dementia, \% & 22.4 & 21.1 & 36.7 & 0.25 \\
\hline Renal failure, \% & 18.4 & 25.5 & 22.4 & 0.29 \\
\hline Diabetes mellitus, \% & 17.5 & 26.7 & 26.5 & 0.150 \\
\hline Cancer, \% & 15.7 & 25.5 & 20.4 & 0.140 \\
\hline Chronic liver disease, \% & 8.1 & 12.2 & 10.2 & 0.55 \\
\hline$>2$ comorbid conditions, $\%$ & 18.4 & 28.9 & 26.5 & 0.080 \\
\hline$\geq 2$ comorbid conditions, \% & 44.4 & 52.2 & 61.2 & $0.030^{*}$ \\
\hline Endotracheal intubation in the past 30 days, $\%$ & 0 & 2.2 & 12.2 & $<0.001^{*}+$ \\
\hline Leukopenia, \% & 3.6 & 4.4 & 12.2 & 0.89 \\
\hline Malnutrition, \% & 4.5 & 11.1 & 16.3 & $0.004^{*} \dagger$ \\
\hline Histamine-2 blockers or antacids, \% & 23.3 & 53.3 & 57.1 & $<0.001^{*}+$ \\
\hline Tracheostomy, \% & 0 & 1.1 & 8.2 & $<0.001^{*}$ \\
\hline Aerosolized drugs, \% & 12.5 & 26.7 & 22.4 & $0.003+$ \\
\hline Previous antibiotic therapy, \% & 32.7 & 38.9 & 42.8 & 0.26 \\
\hline Fever, \% & 62.8 & 45.5 & 49 & $0.020+$ \\
\hline Depression of consciousness, \% & 19.8 & 26.7 & 32.6 & 0.160 \\
\hline Dyspnea, \% & 69 & 70.0 & 49 & 0.060 \\
\hline Cough, \% & 61.3 & 61.1 & 46.9 & 0.180 \\
\hline Purulent sputum, \% & 32 & 36.7 & 34.7 & 0.63 \\
\hline Pleuritic chest pain, \% & 17.1 & 12.2 & 4.1 & 0.60 \\
\hline Leukocytosis, \% & 54.3 & 52.2 & 53.1 & 0.94 \\
\hline Bilateral involvement on chest radiograph, $\%$ & 19.7 & 34.4 & 32.6 & $0.007 *+$ \\
\hline Multilobar infiltrates, \% & 21.5 & 27.8 & 38.8 & $0.020^{*}$ \\
\hline Pleural effusion, \% & 38.7 & 45.5 & 38.8 & 0.56 \\
\hline
\end{tabular}

${ }^{*} P<0.05$ for comparison between hospital-acquired and community-acquired pneumonia.

$+P<0.05$ for comparison between health care-associated and community-acquired pneumonia.

(6.7\% [CI, $2.9 \%$ to $10.5 \%])$ was significantly lower than that for health care-associated pneumonia $(17.8 \%$ [CI, $10.6 \%$ to $24.9 \%$ ]) or hospital-acquired pneumonia (18.4\% [CI, $6.4 \%$ to $30.3 \%]$ ).

In the bivariate analysis, the following factors were significantly associated with in-hospital death: leukopenia, malnutrition, depression of consciousness, pleuritic chest pain, bilateral and multilobar involvement on chest radiography, pleural effusion, inclusion in the health care-associated or hospital-acquired pneumonia group, antibiotic therapy not consistent with recommended guidelines, inappropriate therapy, microbiologic diagnosis, and a Sequential Organ Failure Assessment score greater than 2. When all of the selected variables were put into the multivariate model, 3 factors were independently associated with death: depression of consciousness (OR, 3.2 [CI, 1.06 to 9.8]), leukopenia (OR, 6.2 [CI, 1.01 to 37.6]), and receipt of an empirical antibiotic not recommended by international guidelines (OR, 6.4 [CI, 2.3 to 17.6$])$.

\section{Discussion}

To our knowledge, this is the first prospective, observational, multicenter study that compares the epidemiology and outcome of community-acquired pneumonia, health care-associated pneumonia, and hospital-acquired pneumonia in patients hospitalized in medical wards. Our findings, which may be considered representative of all Italian hospitals, support a new pneumonia classification scheme that distinguishes among community-acquired, health care-associated, and hospital-acquired pneumonia. Compared with community-acquired pneumonia, health care-associated pneumonia is associated with longer hospital stay and higher risk for a fatal complication. Health care-associated pneumonia also differs from hospitalacquired pneumonia that occurs in nonventilated patients; however, in general, health care-associated pneumonia differs from hospital-acquired pneumonia to a lesser degree

$\begin{aligned} & \text { Table 3. Criteria for Inclusion in the Health Care-Associated } \\ & \text { Pneumonia Group }\end{aligned}$
$\begin{array}{ll}\text { Criterion } & \begin{array}{l}\text { Patients } \\ (95 \% \mathrm{Cl}), \% \\ (n=90)\end{array} \\ & 80(75.8-84.2) \\ \text { Recent hospitalization within } 180 \text { days } & 10(6.8-13.2) \\ \text { Nursing home resident } & 3.3(1.4-5.2) \\ \text { Hemodialysis } & 6.7(4.1-9.3) \\ \text { Intravenous chemotherapy in the } 30 \text { days before } \\ \text { pneumonia }\end{array}$


ARTICLE Health Care-Associated Pneumonia as a Distinct Subset of Pneumonia

Table 4. Antimicrobial Therapy and Outcomes

\begin{tabular}{|c|c|c|c|c|}
\hline Variable & $\begin{array}{l}\text { Community-Acquired } \\
\text { Pneumonia }(n=223)\end{array}$ & $\begin{array}{l}\text { Health Care-Associated } \\
\text { Pneumonia }(n=90)\end{array}$ & $\begin{array}{l}\text { Hospital-Acquired } \\
\text { Pneumonia }(n=49)\end{array}$ & $P$ Value \\
\hline Initial antibiotic monotherapy $(95 \% \mathrm{Cl}), \%$ & 54.9 (47.7 to 62.2$)$ & 60.7 (48.1 to 73.2$)$ & $28.6(14.7$ to 42.4$)$ & $0.010^{*}$ \\
\hline Adherence to international guidelines $(95 \% \mathrm{Cl}), \%$ & 58.7 (51.8 to 65.6$)$ & 26.7 (16.5 to 36.8$)$ & 69.4 (57 to 81.8 ) & $<0.001+$ \\
\hline Inappropriate antibiotic therapy $(95 \% \mathrm{CI}), \%$ & 5.8 (2.1 to 9.5$)$ & $18.9(10$ to 27.8$)$ & $10.2(-2.6$ to 23$)$ & $0.020+$ \\
\hline Mean duration of antibiotic therapy $(95 \% \mathrm{Cl}), d$ & $13.8(12.9$ to 14.7$)$ & 16.1 (14.6 to 17.7$)$ & $16.7(14.3$ to 19.1$)$ & $0.010+$ \\
\hline Mean duration of hospitalization $(95 \% \mathrm{Cl}), d$ & $14.7(13.4$ to 15.9$)$ & $18.7(15.9$ to 21.5$)$ & 24.8 (19.8 to 29.8$)$ & $<0.001^{*} \dagger$ \\
\hline Overall in-hospital mortality rate $(95 \% \mathrm{Cl}), \%$ & 6.7 (2.9 to 10.5$)$ & 17.8 (10.6 to 24.9$)$ & $18.4(6.4$ to 30.3$)$ & $0.020+$ \\
\hline
\end{tabular}

${ }^{*} P<0.05$ for comparison between hospital-acquired and community-acquired pneumonia.

$+P<0.05$ for comparison between health care-associated and community-acquired pneumonia.

than from community-acquired pneumonia because the severity of disease and mortality rates are more similar to the former. These results suggest that communityacquired pneumonia, health care-associated pneumonia, and hospital-acquired pneumonia are 3 distinct epidemiologic and clinical entities, with a trend toward increasing disease severity from the first to the last group.

The poor prognosis observed in patients with health care-associated pneumonia seems to be related to greater severity of disease (demonstrated by higher mean Sequential Organ Failure Assessment scores and by more frequent bilateral and multilobar lung involvement). The 3 groups did not significantly differ in terms of mean age or the presence of comorbid conditions. This probably reflects the homogeneity of our sample, because patients hospitalized in internal medicine departments typically have multiple comorbid conditions, higher mean age, and a longer hospital stay than those admitted to other departments (such as oncology or hematology units) or surgical wards. However, patients with health care-associated pneumonia had more comorbid conditions than patients with community-acquired pneumonia (although the differences were not statistically significant, possibly because of sample size limitations); this finding may also explain the higher severity of clinical conditions observed in the health care-associated pneumonia group. Patients with health care-associated pneumonia were also less frequently febrile at admission and were more frequently malnourished and treated with gastric acidsuppressive agents or aerosolized drugs than patients with community-acquired pneumonia. On the other hand, a significantly higher proportion of patients with hospital-acquired pneumonia had a tracheostomy procedure, a history of recent endotracheal intubation, and leukopenia. Overall, patients with hospital-acquired pneumonia seemed the most critically ill.

In Tables 1 and 4, the groups differ significantly for some outcomes, but the CIs of the mean estimates overlap. This is probably because of in-hospital clustering effects. Methods that account for the clustering, such as our bootstrapping procedure, can lead to more efficient estimates of the differences than of the mean values themselves. Thus, the comparisons are robust enough to warrant discussion.
Although the 2005 guidelines from the American Thoracic Society and Infectious Diseases Society of America (3) included specific recommendations for the current management of health care-associated pneumonia, we observed low adherence to available guidelines. Physicians usually do not differentiate between the initial treatment for health care-associated pneumonia and communityacquired pneumonia, and this clinical approach usually results in inappropriate initial therapy and increased mortality. Indeed, regression analysis indicated that administration of empirical antibiotic therapy not recommended in the guidelines was independently associated with increased mortality.

A MEDLINE search of the English-language medical literature using the terms "health care-associated" and "pneumonia" revealed only a few original articles (5-7), most of them retrospective studies $(5,6)$. The definition of health care-associated infections was initially proposed by Friedman and colleagues (2), who chose previous hospitalization within 3 months of the study as the criterion by which to categorize patients as having a health careassociated bloodstream infection. Other investigators extended the criterion of community-onset health careassociated infections to include hospitalization in the preceding 12 months (15). The definition of health careassociated pneumonia has been adapted from the criteria described for bloodstream infections. In the first retrospective study on culture-positive pneumonia conducted in the United States (5), which used a large multi-institutional database, the case definition of health care-associated pneumonia required any of the following criteria: residence in a health care facility, long-term hemodialysis, or hospitalization within 30 days of receiving a diagnosis of pneumonia. In a prospective analysis of patients with communityonset pneumonia requiring hospitalization, Carratalà and colleagues (7) extended the duration criteria of previous hospitalization to 90 days before diagnosis, which is also recommended by the American Thoracic Society and the Infectious Diseases Society of America guidelines (3). In our study, we used 180 days as a cutoff for recent hospitalization to differentiate between community-acquired and health care-associated pneumonia. We based our choice to expand the inclusion criteria for health care- 
associated pneumonia on several considerations, including the prolonged duration of colonization and possible subsequent infections with resistant bacteria (such as Staphylococcus aureus) after hospital discharge (16). The importance of considering prolonged bacterial colonization was also suggested by a retrospective cohort study (6) involving 639 patients with culture-positive community-acquired and health care-associated pneumonia who were admitted to a single center. This study revealed that patients who were hospitalized for 2 or more days in the preceding 12 months were also at risk for multidrug-resistant pathogens. Our findings confirm that a window of 180 days for recent hospitalization may be useful to clearly differentiate between community-acquired and health care-associated pneumonia (17).

Although the available studies (5-7) concur that health care-associated pneumonia is a distinctive category of lower respiratory tract infection, they differ in important ways. The reported incidence of health care-associated pneumonia among patients requiring hospitalization ranged from $17.3 \%$ to $67.4 \%$, depending on the sample analyzed and the study methods used (5-7). In our sample, for which we used the extended criteria for recent hospitalization, health care-associated pneumonia accounted for $28.7 \%$ of all cases of community-onset pneumonia. Incidence of health care-associated pneumonia may be underestimated because of the lack of documentation at hospital admission of various criteria that fulfill the definition of health care-associated pneumonia. Data concerning cause are also variable. In a large retrospective study of 4543 patients with culture-positive pneumonia (5), Kollef and colleagues found that $S$. aureus was a major pathogen $(46.7 \%)$ in health care-associated pneumonia, followed by $P$. aeruginosa $(25.3 \%)$, Klebsiella species $(7.6 \%)$, and others. In Carratalà and colleagues' study (7), the most frequent causative organism in health care-associated pneumonia and community-acquired pneumonia was Streptococcus pneumoniae, which accounted for $27.8 \%$ and $33.9 \%$ of cases, respectively. However, S. aureus and gram-negative bacilli were significantly more frequent in health care-associated pneumonia than in community-acquired pneumonia $(P=0.005$ and $P=$ 0.03 , respectively). These observations may explain the failure of antibiotic regimens that target the usual community-acquired pneumonia pathogens, such as $S$. pneumoniae, Haemophilus influenzae, or Mycoplasma pneumoniae. However, the role of resistant pathogens in health care-associated pneumonia needs to be addressed by future studies.

Our study has several limitations. First, it was a pragmatic study that included both tertiary and secondary care centers. Microbiological investigation was therefore neither homogeneous nor extensively performed in all cases. Second, the number of patients with health care-associated pneumonia was relatively small. Finally, we included only patients with pneumonia that required hospitalization; thus, our results may not apply to patients treated as outpatients. Future prospective studies are needed to clarify the microbiology of health care-associated pneumonia in hospitalized patients. Moreover, the importance of the new pneumonia classification will rest on randomized and observational studies that demonstrate improved outcomes for patients with health care-associated pneumonia managed with guidelines specific to health care-associated pneumonia.

Our data provide further evidence that health careassociated pneumonia is usually not recognized or managed correctly by clinicians, possibly resulting in inappropriate antibiotic therapy and increased mortality. The selection of empirical antibiotics for health care-associated pneumonia should include coverage of methicillin-resistant $S$. aureus and multidrug-resistant gram-negative rods (such as linezolid or glycopeptides plus an antipseudomonal $\beta$-lactam), as recommended by the consensus of Infectious Diseases Society of America experts (18).

In conclusion, health care-associated pneumonia represents a distinct subset of pneumonia. Compared with patients who have community-acquired pneumonia, patients with health care-associated pneumonia have more severe disease, prolonged hospitalization, and increased mortality. Consequently, health care-associated pneumonia is more similar to hospital-acquired pneumonia than community-acquired pneumonia and requires specific initial empirical antibiotic therapy. Physicians should differentiate between patients with health care-associated pneumonia and those with community-acquired pneumonia to provide optimal clinical management.

From the University of Rome, Rome, Italy, and Università degli Studi di Palermo, Palermo, Italy.

Acknowledgment: The authors thank Professor Pier Mannuccio Mannucci, Milan, Italy, for his activity as Chief of the Italian Society of Internal Medicine group for independent clinical research. They also thank Sohita Dhillon and Rod McNab of Wolters Kluwer Health for English-language assistance in the preparation of this manuscript.

Potential Financial Conflicts of Interest: Consultancies: M. Venditti (Glaxo Wellcome, Gilead, Angelini, Novartis, Pfizer, Bayer, Wyeth). Grants received: M. Falcone (Pfizer).

Reproducible Research Statement: Study protocol: Available at www .simi.it. Statistical code: Available from Dr. Corrao (s.corrao@unipa.it). Data set: Not available.

Requests for Single Reprints: Mario Venditti, MD, Dipartimento di Medicina Clinica-Policlinico Umberto I, Università di Roma "La Sapienza," Viale dell'Università 37, 00161 Rome, Italy; e-mail, mario .venditti@uniroma1.it.

Current author addresses and author contributions are available at www .annals.org.

6 January 2009 $\mid$ Annals of Internal Medicine $\mid$ Volume 150 • Number $1 \mid 25$ 
ARTICLE $\mid$ Health Care-Associated Pneumonia as a Distinct Subset of Pneumonia

\section{References}

1. Marrie TJ, Wu L. Factors influencing in-hospital mortality in communityacquired pneumonia: a prospective study of patients not initially admitted to the ICU. Chest. 2005;127:1260-70. [PMID: 15821203]

2. Friedman ND, Kaye KS, Stout JE, McGarry SA, Trivette SL, Briggs JP, et al. Health care-associated bloodstream infections in adults: a reason to change the accepted definition of community-acquired infections. Ann Intern Med. 2002;137:791-7. [PMID: 12435215]

3. American Thoracic Society, Infectious Diseases Society of America. Guidelines for the management of adults with hospital-acquired, ventilator-associated, and healthcare-associated pneumonia. Am J Respir Crit Care Med. 2005;171: 388-416. [PMID: 15699079]

4. Tablan OC, Anderson LJ, Besser R, Bridges C, Hajjeh R, for the Healthcare Infection Control Practices Advisory Committee. CDC. Guidelines for preventing health-care-associated pneumonia, 2003: recommendations of CDC and the Healthcare Infection Control Practices Advisory Committee. MMWR Recomm Rep. 2004;53:1-36. [PMID: 15048056]

5. Kollef MH, Shorr A, Tabak YP, Gupta V, Liu LZ, Johannes RS. Epidemiology and outcomes of health-care-associated pneumonia: results from a large US database of culture-positive pneumonia. Chest. 2005;128:3854-62. [PMID: 16354854]

6. Micek ST, Kollef KE, Reichley RM, Roubinian N, Kollef MH. Health care-associated pneumonia and community-acquired pneumonia: a singlecenter experience. Antimicrob Agents Chemother. 2007;51:3568-73. [PMID: 17682100]

7. Carratalà J, Mykietiuk A, Fernández-Sabé N, Suárez C, Dorca J, Verdaguer $\mathrm{R}$, et al. Health care-associated pneumonia requiring hospital admission: epidemiology, antibiotic therapy, and clinical outcomes. Arch Intern Med. 2007;167: 1393-9. [PMID: 17620533]

8. Fine MJ, Auble TE, Yealy DM, Hanusa BH, Weissfeld LA, Singer DE, et al. A prediction rule to identify low-risk patients with community-acquired pneumonia. N Engl J Med. 1997;336:243-50. [PMID: 8995086]

9. Lim WS, van der Eerden MM, Laing R, Boersma WG, Karalus N, Town GI, et al. Defining community acquired pneumonia severity on presentation to hospital: an international derivation and validation study. Thorax. 2003;58:37782. [PMID: 12728155]
10. Physical Status: The Use and Interpretation of Anthropometry. World Health Organization Technical Report Series 854. Geneva: World Health Organization; 1995. Accessed at whqlibdoc.who.int/trs/WHO_TRS_854.pdf on 24 October 2008.

11. Mykietiuk A, Carratalà J, Domínguez A, Manzur A, Fernández-Sabé N, Dorca J, et al. Effect of prior pneumococcal vaccination on clinical outcome of hospitalized adults with community-acquired pneumococcal pneumonia. Eur J Clin Microbiol Infect Dis. 2006;25:457-62. [PMID: 16773389]

12. Mandell LA, Wunderink RG, Anzueto A, Bartlett JG, Campbell GD, Dean NC, et al. Infectious Diseases Society of America. Infectious Diseases Society of America/American Thoracic Society consensus guidelines on the management of community-acquired pneumonia in adults. Clin Infect Dis. 2007;44 Suppl 2:S27-72. [PMID: 17278083]

13. Arancibia F, Bauer TT, Ewig S, Mensa J, Gonzalez J, Niederman MS, et al. Community-acquired pneumonia due to gram-negative bacteria and Pseudomonas aeruginosa: incidence, risk, and prognosis. Arch Intern Med. 2002;162:184958. [PMID: 12196083]

14. Hosmer DW, Lemeshow S. Applied Logistic Regression. New York: J Wiley; 2002.

15. Morin CA, Hadler JL. Population-based incidence and characteristics of community-onset Staphylococcus aureus infections with bacteremia in 4 metropolitan Connecticut areas, 1998. J Infect Dis. 2001;184:1029-34. [PMID: 11574918]

16. Scanvic A, Denic L, Gaillon S, Giry P, Andremont A, Lucet JC. Duration of colonization by methicillin-resistant Staphylococcus aureus after hospital discharge and risk factors for prolonged carriage. Clin Infect Dis. 2001;32:1393-8. [PMID: 11317238]

17. Abrahamian FM, Deblieux PM, Emerman CL, Kollef MH, Kupersmith E, Leeper KV Jr, et al. Health care-associated pneumonia: identification and initial management in the ED. Am J Emerg Med. 2008;26:1-11. [PMID: 18603170] 18. Kollef MH, Morrow LE, Baughman RP, Craven DE, McGowan JE Jr, Micek ST, et al. Health care-associated pneumonia (HCAP): a critical appraisal to improve identification, management, and outcomes-proceedings of the HCAP Summit. Clin Infect Dis. 2008;46 Suppl 4:S296-334; quiz 335-8. [PMID: 18429676]

\section{New Peer Reviewers}

Sign up to become a peer reviewer for Annals of Internal Medicine by going to www.annals.org and selecting "Information for: Authors/ Reviewers." Then select "Reviewer Information" and register as a new reviewer. Note that Annals reviewers whose reviews are returned on time and are judged satisfactory by the Editors may receive up to 3 Category 1 CME credits per review (maximum, 15 credits in a calendar year). 
Current Author Addresses: Drs. Venditti, Falcone, and Serra: Dipartimento di Medicina Clinica-Policlinico Umberto I, Università di Roma "La Sapienza," Viale dell’Università 37, 00161 Rome, Italy.

Drs. Corrao and Licata: Dipartimento Biomedico di Medicina Interna e Specialistica, Università degli Studi di Palermo, Piazza delle Cliniche 2, 90127 Palermo, Italy.

Author Contributions: Conception and design: M. Venditti, M. Falcone, P. Serra.

Analysis and interpretation of the data: M. Venditti, M. Falcone, S. Corrao.

Drafting of the article: M. Venditti, M. Falcone.

Critical revision of the article for important intellectual content: S. Corrao, G. Licata, P. Serra.

Final approval of the article: S. Corrao, G. Licata, P. Serra.

Provision of study materials or patients: G. Licata.

Statistical expertise: S. Corrao.

Obtaining of funding: G. Licata.

Administrative, technical, or logistic support: G. Licata.

Collection and assembly of data: M. Falcone, S. Corrao.

\section{Appendix: Participating Members of the Italian Society of Internal Medicine}

Medicina Interna, Policlinico San Donato Milanese:

Francesco Salerno.

Dipartimento di Scienze Cliniche, Policlinico Umberto IUniversità di Roma "La Sapienza": Sebastiano Filetti, Emilio D'Erasmo.

II Patologia Medica, Policlinico Umberto I-Università di Roma "La Sapienza": Filippo Rossi Fanelli.

Geriatria, Ospedale Civile, Montefiascone: Alessandra Fiorentini, Luigi Cricco.

Medicina Interna 5, Azienda Ospedaliera San CamilloForlanini, Roma: Laura Gasbarrone, Cecilia Serafini.

Clinica Medicina Interna 3, Azienda Ospedaliera Universitaria San Martino, Genova: Riccardo Ghio, Gabriele Zoppoli.

Medicina Generale III, Ospedale Luigi Sacco, Milano: Michele Cortellaro, Marina Magenta.

Medicina Generale I, Azienda Ospedaliera Universita degli Studi di Siena: Ranuccio Nuti, Roberto Valenti.

Presidio Ospedaliero C. Ondoli, Angera: Vincenzo Milano.

Medicina Interna, Azienda Ospedaliera San Giovanni Addolorata, Roma: Camillo Brandimarte, Paolo Carfagna.

Divisione Medicina Interna e Specialistica, Policlinico Universitario, Palermo: Riccardo Di Sciacca, Antonino Tuttolomondo.

Unità Operativa Medicina, Azienda Ospedaliera Cardinale G. Panico, Tricase: Maria Grazia Serra.

Semeiotica Medica, Policlinico S. Orsola Malpighi, Bologna: Mauro Bernardi, Silvia Li Bassi.

Dipartimento Medicina Interna e Gastroenterologia, Policlinico S. Orsola Malpighi, Bologna: Vincenzo Stanghellini, Elena Boschi.

Medicina Interna Universitaria "Cesare Frugoni", Policlinico, Bari: Salvatore Antonaci, Francesco Vella.

Dipartimento di Medicina Interna-Policlinico Universitario "G. Martino" di Messina: Antonino Catalano.

Divisione Medicina, Azienda Ospedaliera Universitaria, Modena: Maria Luisa Zeneroli, Elisabetta Ascari, Alberto Veggetti.
Ospedale del Delta, Lagosanto: Roberto Manfredini, Susanna Gamberoni.

Istituto di Clinica Medica Generale, Ospedale di Cattinara, Trieste: Gianfranco Guarnieri, Angela Fioretto.

Medicina Interna, Ospedale "G. Mazzini" ASL Teramo: Dario Di Michele, Domenico Parisi.

Divisione di Medicina Interna, Ospedale Civile, Casorate Primo: Nicola Lucio Liberato, Esio Ronchi.

Medicina Interna, Ospedale Civile, Senigallia: Simonetta Sturbini, Paolo Canafoglia.

Medicina Interna Ospedaliera, Azienda Ospedaliera Universitaria Sant'Anna, Ferrara: Massimo Gallerani, Benedetta Boari.

Medicina ad Alta Rotazione, Azienda Ospedaliera Universitaria Sant'Anna, Ferrara: Ingrid Nielsen.

Geriatria, Azienda Ospedaliera San Gerardo, Monza: Giorgio Annoni, Anna Rossetti.

Azienda Ospedaliera Ospedale di Circolo Fondazione Macchi, Varese: Matteo Bernasconi.

Medicina Interna, Azienda Ospedaliera Policlinico Bari: Carmela Giannatempo.

Medicina I, Azienda Ospedaliera G. Salvini, Garbagnate Milanese: Roberta Turconi, Maurizio Colombo.

Medicina Interna 2, Policlinico Ospedale Maggiore, Milano: Alberto Tedeschi, Raffaella Rossio.

Medicina Interna II, Policlinico S. Maria alle Scotte, Siena: Roberto Cappelli, Valentina Guidi.

Medicina 1 A.S.L. 2 Savonese Ospedale San Paolo, Savona: Rodolfo Tassara, D. De Melis.

Medicina Interna, Fondazione IRCCS Ospedale Maggiore Policlinico, Mangiagalli e Regina Elena, Milano: Roberto Cosentini, Margherita Arioli, Francesco Salerno, Giulia Gobbo.

Medicina Generale, A.S.L. 17, Ospedale di Este: Fabio Presotto, Sergio Gallana.

Clinica Medica III, Fondazione IRCCS Policlinico San MatteoUniversità di Pavia: Carlo Balduini, Giampiero Bertolino.

Medicina Interna, Presidio Ospedaliero-A.S.L. BA/5, Monopoli-Conversano: Giacomo Fera.

Clinica Medica I, Policlinico San Matteo, Pavia: Gino Roberto Corazza, Ida Capriglione.

Medicina, Presidio Sanitario Gradenigo, Torino: Giulia Pilerio.

Dipartimento di Medicina Interna-Fondazione IRCCS Ospedale Maggiore Policlinico, Mangiagalli e Regina Elena \& Università degli studi di Milano: Maria Domenica Cappellini, Giovanna Fabio, Maria Carrabba.

Medicina Generale, Ospedale E. Bassini, Cinisello Balsamo, Azienda Ospedaliera S. Gerardo, Monza: Sheng Chin Wu, Maria Beatrice Secchi.

Medicina, Casa di Cura La Madonnina, Bari: Michele Leone.

Medicina Interna, Presidio Ospedaliero, Pescara: Lucrezia De Feudis.

Medicina Interna Ospedale San Salvatore Pesaro: Massimo Gunelli, Orazio Ferri.

Medicina Interna 2, Presidio Ospedaliero Cà Fondello, Treviso: Carlo Doroldi, Roberta Pistis.

Università Vita-Salute San Raffaele-Milano: Maria Grazia Sabbadini, Moreno Tresoldi.

6 January 2009 Annals of Internal Medicine $\mid$ Volume 150 • Number $1 \mid \mathbf{W}-3$ 
Medicina Interna 5, Ospedale Unico della Versilia, Lido di Camaiore: Paola Lambelet, Stefano Fascetti.

Struttura Complessa di Medicina Interna, A.O. Ospedale di Lecco, P.O. di Merate: Massimo Vanoli, Gianluca Casella.

Clinica Medica Generale, Azienda Ospedali Civili, Brescia: Enrico Agabiti Rosei, Andrea Salvi.

Medicina Interna, Ospedale Civile dell'Annunziata, Cosenza: Alfonso Noto.

Medicina Interna, Ospedale San Giovanni DecollatoAndosilla, Cività Castellana: Antonio Perciaccante.

Medicina Interna, Ospedale Madre G. Vannini, Roma: Claudio Santini, Maurizia Galiè.
Istituto di Medicina Interna e Geriatria, Università Cattolica del Sacro Cuore, Policlinico A. Gemelli, Roma: Giovanni Gasbarrini, Antonio Grieco.

Medicina 1, Azienda Ospedaliera di Busto Arsizio (VA) Ospedale di Circolo di Busto Arsizio: Barbara Nardi.

Clinica Medica 1, Azienda Ospedaliera, Padova: Aldo Gaetano Baritussio, Roberto Vannuccini.

Medicina Generale, Alessandria: Mauro Cappelletti.

Medicina d'Urgenza e Pronto Soccorso, Università Cattolica del Sacro Cuore, Policlinico A. Gemelli, Roma: Nicolò GentiloniSilveri.

Medicina Interna C, Policlinico G.B. Rossi, Verona: Alessandro Lechi, Germana Montesi. 
Appendix Table 1. Risk Stratification (Incident Cases Only)

Variable

Mean PSI score

PSI high-risk class*

CURB-65 high-risk scoret
Community-Acquired Pneumonia $(95 \% \mathrm{Cl})$, \%

$(n=97)$

$105.9(98.6-113.2)$

$68.0(58.7-77.4)$

$11.3(4.5-18.2)$
Health Care-Associated

Pneumonia $(95 \% \mathrm{Cl})$, \%

( $n=31)$

$122.0(112-132)$

$97.8(90.5-100)$

$32.2(16.3-48.2)$
$P$ Value

0.010

$<0.001$

0.090

CURB-65 = Confusion, Urea nitrogen, Respiratory rate, Blood pressure, and age 65 years or older; PSI = Pneumonia Severity Index.

* Class IV or V (8).

† Class III (9).

\section{Appendix Table 2. Patient Characteristics (Incident Cases Only)}

\begin{tabular}{|c|c|c|c|c|}
\hline Characteristic & $\begin{array}{l}\text { Community-Acquired } \\
\text { Pneumonia }(n=97)\end{array}$ & $\begin{array}{l}\text { Health Care-Associated } \\
\text { Pneumonia }(n=31)\end{array}$ & $\begin{array}{l}\text { Hospital-Acquired } \\
\text { Pneumonia }(n=16)\end{array}$ & $P$ Value \\
\hline Men, \% & 61.8 & 64.5 & 68.7 & 0.89 \\
\hline Mean age, $y$ & 70.8 & 77.4 & 79.7 & 0.28 \\
\hline Chronic obstructive pulmonary disease, \% & 42.3 & 41.9 & 25 & 0.45 \\
\hline Heart failure, \% & 22.7 & 38.7 & 18.7 & 0.190 \\
\hline Dementia, \% & 22.7 & 16.1 & 37.5 & 0.25 \\
\hline Renal failure, \% & 14.4 & 29 & 18.7 & 0.170 \\
\hline Diabetes mellitus, \% & 15.5 & 22.6 & 25 & 0.47 \\
\hline Cancer, \% & 14.4 & 32.2 & 25 & 0.070 \\
\hline Chronic liver disease, $\%$ & 9.3 & 12.9 & 12.5 & 0.69 \\
\hline$>2$ comorbid conditions, $\%$ & 14.4 & 32.2 & 31.2 & 0.030 \\
\hline$\geq 2$ comorbid conditions, $\%$ & 42.3 & 54.8 & 62.5 & 0.22 \\
\hline Endotracheal intubation in the past 30 days, \% & 0 & 3.2 & 12.5 & 0.009 \\
\hline Leukopenia, \% & 4.1 & 3.2 & 6.2 & 0.82 \\
\hline Malnutrition, \% & 6.2 & 16.1 & 25 & 0.020 \\
\hline Histamine-2 blockers or antacids, $\%$ & 20.6 & 48.4 & 68.7 & $<0.001$ \\
\hline Tracheostomy, \% & 0 & 3.2 & 12.5 & 0.009 \\
\hline Aerosolized drugs, \% & 15.5 & 25.8 & 25 & 0.29 \\
\hline Previous antibiotic therapy, \% & 33 & 38.7 & 50 & 0.41 \\
\hline Fever, \% & 62.9 & 38.7 & 56.2 & 0.060 \\
\hline Depression of consciousness, \% & 17.5 & 35.5 & 43.7 & 0.010 \\
\hline Dyspnea, \% & 68 & 77.7 & 50 & 0.38 \\
\hline Cough, \% & 57.7 & 48.4 & 43.7 & 0.45 \\
\hline Purulent sputum, \% & 30.9 & 38.7 & 25 & 0.62 \\
\hline Pleuritic chest pain, \% & 20.6 & 6.4 & 6.2 & 0.100 \\
\hline Leukocytosis, \% & 63.9 & 48.4 & 56.2 & 0.25 \\
\hline Bilateral involvement on chest radiograph, \% & 16.5 & 45.2 & 25 & 0.005 \\
\hline Multilobar infiltrates, \% & 22.7 & 25.8 & 43.7 & 0.21 \\
\hline Pleural effusion, \% & 42.3 & 38.7 & 37.5 & 0.90 \\
\hline Mean SOFA score & 1.84 & 3.38 & 3.37 & $<0.001$ \\
\hline Microbiological diagnosis, \% & 15.5 & 35.5 & 18.7 & 0.060 \\
\hline
\end{tabular}

SOFA $=$ Sequential Organ Failure Assessment.

Appendix Table 3. Antimicrobial Therapy and Outcomes (Incident Cases Only)

Variable

Initial antibiotic monotherapy $(95 \% \mathrm{Cl}), \%$

Adherence to international guidelines ( $95 \% \mathrm{Cl}), \%$

Inappropriate antibiotic therapy $(95 \% \mathrm{Cl}), \%$

Mean duration of antibiotic therapy $(95 \% \mathrm{Cl}), d$

Mean duration of hospitalization $(95 \% \mathrm{Cl}), d$

Overall in-hospital mortality rate $(95 \% \mathrm{Cl}), \%$
Community-Acquired
Pneumonia $(n=97)$

53.1 (41.7 to 64.5)

$59.8(50.3$ to 69.3$)$

$4.1(-0.3$ to 8.5$)$

$12.8(11.5$ to 14.1$)$

12.5 (11 to 13.9$)$

7.2 (2.4 to 12$)$
Health Care-Associated

Pneumonia $(n=31$ )

66.7 (49.1 to 84.2)

35.5 (18.4 to 52.6 )

16.1 (0 to 32.2)

14.4 (11.9 to 16.9$)$

15.9 (10.6 to 21.1 )

22.6 (9.6 to 35.5 )
Hospital-Acquired Pneumonia $(n=16)$

43.7 (21.8 to 65.7)

50 (27.8 to 72.2$)$

$6.2(-3.8$ to 16.3$)$

11.4 (8.8 to 14 )

14.7 (10.1 to 19.3$)$

18.7 ( -2.2 to 39.7$)$
$P$ Value 